\title{
Medial Axis Seeding of a Guided Evolutionary Simulated Annealing (GESA) Algorithm for Automated Gamma Knife Radiosurgery Treatment Planning
}

\author{
David Dean ${ }^{1}$, Pengpeng Zhang ${ }^{2}$, Andrew K. Metzger ${ }^{1}$, Claudio Sibata ${ }^{3}$, and \\ Robert J. Maciunas ${ }^{1}$ \\ ${ }^{1}$ Department of Neurological Surgery, and The Research Institute, University Hospitals of \\ Cleveland, and Department of Neurological Surgery, Case Western Reserve University \\ 10900 Euclid Avenue, Cleveland, OH 44106-5042 USA \\ $\{d x d 35, r j m 31, a k m 8\} @ p o . c w r u . e d u$ \\ ${ }^{2}$ Department of Biomedical Engineering, Case Western Reserve University \\ 10900 Euclid Avenue, Cleveland, OH 44106-7207 USA \\ pxz11@po.cwru.edu \\ ${ }^{3}$ Department of Radiation Oncology, and The Research Institute, University Hospitals of \\ Cleveland, and Department of Radiation Oncology, Case Western Reserve University \\ 10900 Euclid Avenue, Cleveland, OH 44106-6068 USA \\ cxs81@po.cwru.edu
}

\begin{abstract}
We present a method to optimize Gamma Knife ${ }^{\mathrm{TM}}$ (Elekta, Stockholm, Sweden) radiosurgery treatment planning. A Guided Evolutionary Simulated Annealing optimization algorithm is used to maximize the therapeutic benefit through a probability model that dissects a patient volume image into three components: normal, critical normal, and tumor tissue. This evolutionary optimization algorithm may be seeded randomly or via an automatically detected medial axis. We use indices of dose conformality, level, and homogeneity to evaluate the degree to which a treatment plan has been optimized. Two clinical examples compare the GESA algorithm with current manual methods. GESA optimization shows therapeutic advantage over the treatment team's manual effort. We find that computation of treatment plans with more than 8 shots require initial medial axis seeding (i.e., shot: number, size, and position) to complete within 8 hours on our workstation.
\end{abstract}

\section{Introduction}

The Leksell Gamma Knife ${ }^{\mathrm{TM}}$ (LGK) is a tool for providing highly accurate stereotactic radiosurgical treatment of brain tumors. It conforms radiation beams to a lesion from $201{ }^{60} \mathrm{Co}$ sources through four different size collimators. Typically, a neurosurgeon, a radiation oncologist, and a medical physicist collaborate to form a unique treatment plan for each patient. Each application of radiation, a "shot," has an ellipsoid shape dose distribution that varies with the location of the isocenter. Typically, more than one shot is usually required to irradiate the tumor. The clinicians provide each shot in the plan to the stationary patient as a separate procedure. 
The tumor portion, and perhaps nearby critical structures, are most often segmented (labeled) in a series of 2D MRI images. The planning team prescribes a radiation dose $\left(\mathrm{D}_{\text {pd }}\right.$, usually $50 \%$ of $\left.\mathrm{D}_{\text {pmax }}\right)$ based in large part on tumor type and volume. Now the number of shots, shot size (only four radiation collimators are available, with diameters of $4 \mathrm{~mm}, 8 \mathrm{~mm}, 14 \mathrm{~mm}$, or $18 \mathrm{~mm}$ respectively), shot weight (irradiation level), and shot position are determined.

Treatment parameters which a computer is better suited to optimize than the treatment team are best handled through inverse-planning. These tasks include determining the location of shots which best conform to, and provide homogeneous irradiation to, the lesion at a prescribed level. Highly conformal plans also contribute to minimization of normal tissue damage. The shot positioning problem can be viewed as a combinatorial packing problem; a search for a group of roughly $3 \mathrm{D}$ Gaussian functions (shots) that conform to the 3D tumor shape under the constraint of minimized spread out to the adjacent volume. However, the limited number of LGK shot sizes and weights (dose level) require complex compromise between the goal of irradiating tumor and sparing normal tissue. The physician specifies where critical structures next to the tumor demand a rapid dose drop-off (steep gradient).

When multiple shots are applied in the treatment plan, the dose is normalized to the maximum dose in the Volume of Interest (VOI). For a treatment plan that includes $\mathrm{N}_{\mathrm{s}}$ shots, each with weight $\mathrm{w}_{\mathrm{s}}$, size $\mathrm{r}$, iscocenter $(\mathrm{i}, \mathrm{j}, \mathrm{k})$, the dose delivered to the voxel $\mathrm{A}$ in the VOI, can be written as:

$$
D_{A}=\frac{\sum_{s=1}^{N_{s}} D_{s A}(w, i, j, k, r)}{\max \left\{\sum_{s=1}^{N_{s}} D_{s B}(w, i, j, k, r), \quad \forall B \in V O I\right\}} \times D_{p \max }
$$

where $D_{\text {pmax }}$ is the prescribed maximum dose by the treatment planning team, and $\mathrm{D}_{\mathrm{sA}}(\mathrm{w}, \mathrm{i}, \mathrm{j}, \mathrm{k}, \mathrm{r})$ is dose contributed to point A via shot $\mathrm{s}$. The goal is to maximize tumor are covered with greater than $50 \%$ of $\mathrm{D}_{\text {pmax }}$, and minimize the normal tissue damage by delivering less than $50 \%$ of $\mathrm{D}_{\text {pmax }}$ there, much less to adjacent critical structures.

Elekta's LGK treatment planning software, GammaPlan ${ }^{\mathrm{TM}}$, asks the treatment planning team to manually choose shot size, weight, and position based on the patient's 2D MR-scan series. The team re-computes the cumulative dose and dose distribution as each new shot is added to the treatment plan, until a sufficient dose acceptably conforms to the lesion. In our experience this iterative procedure ranges from 45 minutes to over 5 hours.

\subsection{Automated Radiosurgery Planning}

Several other groups have presented inverse planning of LGK treatment variables. Shu et. al. ${ }^{1}$ present a solution the shot packing problem that uses multiplier penalty methods. Gibon et al. ${ }^{2}$ solve the same LGK shot positioning problem with a conjugate gradient and simulated annealing approach. Wu et al. ${ }^{3}$ and Wagner et al. ${ }^{4}$ treat LINAC radiosurgery planning as a "shot packing" problem using a depth map similar to our medial axis seeding algorithm. Leichtman et al. ${ }^{5}$ use an adaptive simulated annealing method to refine an initial plan generated automatically by computer or manually by 
the physician. Our Guided Evolutionary Simulated Annealing (GESA) algorithm ${ }^{6}$ hybridizes the evolutionary nature of genetic algorithms with parallel computing (i.e., parallel processing of competing treatment plans), and objective function optimization procedures. The optimized objective function represents a biological model of all treatment criteria. This algorithm discovers a near-optimal solution after examining a small fraction of the possible solutions. ${ }^{7}$

\section{Treatment Criteria Model}

Our mathematical model simulates all treatment criteria. For each $\mathrm{ijk} \mathrm{k}^{\text {th }}$ voxel in a VOI identified by the surgeon. The therapeutic benefit is modeled by probability $\mathrm{P}^{+}{ }_{\mathrm{ijk}}$ :

$$
P_{i j k}^{+}=\frac{e^{K_{i j k} \times \Delta d_{i j k}}}{e^{K_{i j k} \times \Delta d_{i j k}}+e^{-K_{i j k} \times \Delta d_{i j k}}}
$$

where $\Delta \mathrm{d}_{\mathrm{ijk}}=\left(\mathrm{D}_{\mathrm{ijk}}-\mathrm{D}_{\mathrm{PD}}\right) / \mathrm{D}_{\mathrm{PD}}, \mathrm{D}_{\mathrm{ijk}}$ is the dose delivered to the $\mathrm{ijk} \mathrm{k}^{\text {th }}$ voxel; $\mathrm{D}_{\mathrm{pd}}$ is assigned as $50 \%$ of $\mathrm{D}_{\text {pmax }}$; and $\mathrm{K}_{\mathrm{ijk}}$ is a scale factor (imposing the importance of fitting the desired dose to the voxel). Our goal is to maximize therapeutic benefit (i.e., tumor destruction and normal tissue sparing). For a voxel classified as tumor, the associated $\mathrm{K}_{\mathrm{ijk}}$ is positive. Dose that is between the prescribed and maximum levels results in a large $\mathrm{P}^{+}{ }_{\mathrm{ijk}}$. For a voxel classified as normal tissue, the associated $\mathrm{K}_{\mathrm{ijk}}$ is negative. Normal tissue dose below the prescribed minimum tumor level results in a beneficial (large) $\mathrm{P}^{+}{ }_{\mathrm{ijk}}$. For a voxel classified as critical structure, the associated $\mathrm{K}_{\mathrm{ijk}}$ is yet more negative than other normal tissue; the corresponding $\mathrm{D}_{\mathrm{pd}}$ would be assigned an even lower value (i.e., $30 \%$ of $\mathrm{D}_{\text {pmax }}$ ) relative to the prescribed tumor dose. Therefore, a large dose (compared to its own $\mathrm{D}_{\mathrm{pd}}$ ) delivered to critical structure voxels will result in a very small $\mathrm{P}^{+}{ }_{\mathrm{ijk}}$ value, counter to our goal of maximizing $\mathrm{P}^{+}{ }_{\mathrm{ijk}}$. The choice of each $\mathrm{K}_{\mathrm{ijk}}$, especially the ratio different tissue type $\mathrm{K}_{\mathrm{ijk}}{ }^{6} \mathrm{~s}$, determines the compromise between irradiating the entire tumor and sparing adjacent normal tissue.

\section{Objective Function}

An objective function is used to achieve the goal of optimizing the initial plan to match the clinician-specified treatment criteria. As noted, the goal of our objective function is to maximize the plan's therapeutic benefit, modeled as a probability, $\mathrm{P}^{+}{ }_{\mathrm{ijk}}$, at each voxel. The objective function to be minimized (i.e., annealed) is written as:

$$
E=-\frac{1}{N} \sum_{i j k}\left(P_{i j k}^{+}\right)^{2}
$$

where $\mathrm{N}$ is the number of voxels in the VOI. Maximizing $\mathrm{P}^{+}{ }_{\mathrm{ijk}}$ also works towards another planning goal, homogeneous dose distribution (i.e., no hot or cold spots). 


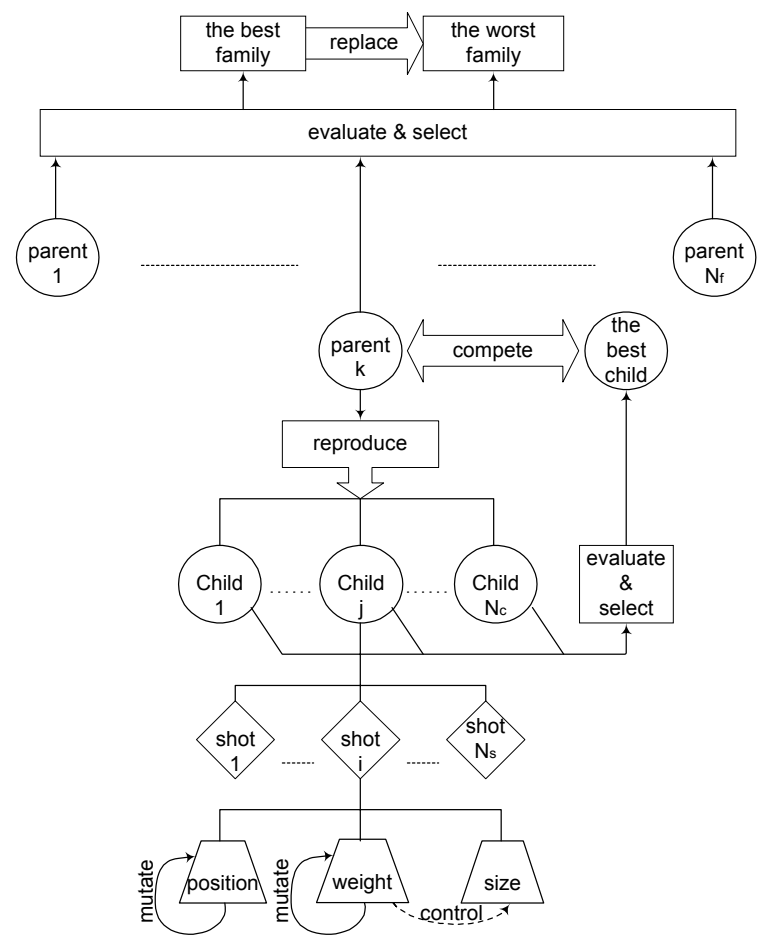

Fig. 1. GESA Evolutionary Procedure. See text for explanation.

\subsection{GESA Optimizes Objective Function}

The GESA algorithm (Figure 1) begins by first choosing $\mathrm{N}_{\mathrm{p}}$ parents from the search space. Each parent generates $\mathrm{N}_{\mathrm{c}}$ children. There is a small random mutation between the parent and its children. The child is accepted only if it satisfies:

$e^{-\left|x_{c}-x_{p}\right| / T_{3}}>p$

where $x_{c}$ is the child, $x_{p}$ is the parent, $T_{3}$ is a temperature parameter, and $\mathrm{p}$ is random between 0 and 1 .

There are two levels of competition in our GESA algorithm. First, within a family, children compete with their parents for a position as a parent in the next generation. The objective function value of the best child in the family is $\mathrm{y}_{\mathrm{bc}}, \mathrm{y}_{\mathrm{p}}$ is the objective function value of the parent, $\mathrm{T}_{1}$ is a temperature parameter, and $\mathrm{p}$ is a random number. The best child in the family is qualified to replace its parent if it satisfies:

$$
y_{b c}<y_{p} \quad \text { or } \quad e^{\left(y_{b c}-y_{p}\right) / T_{1}}>p
$$

Second, there is also competition among families. We evaluate the quality of each family. There are different criteria for inter-family competition.

There are three temperature parameters for the optimization procedure, $\mathrm{T}_{1}, \mathrm{~T}_{2}$, and $\mathrm{T}_{3}$. Between successive generations, the temperature decreases linearly:

$$
T(n)=T(0)-n(T(N)-T(0)) / N
$$

where $\mathrm{T}(0)$ is the initial temperature, $\mathrm{N}$ is the designed maximum generations, and $\mathrm{T}(\mathrm{N})$ is the final temperature.

\section{Seeding the GESA Radiosurgery Treatment Planning Algorithm}

Since the discrete shot number and size are not fixed during the optimization process, there is evolutionary mutation of these parameters in the GESA procedure. Evolution requires variation, therefore we seed GESA with multiple species (i.e., initial treatment plans with different shot number and size) and let them compete for 
survival. For different levels of tumor complexity (i.e., size and geometry), the optimization task utilizes different GESA strategies. For a small tumor with regular shape (i.e., requiring less than 8 shots to cover the tumor), the GESA algorithm begins with a randomly determined initial plan with a maximum number of shots of uniform size by the treatment planning team. From this starting point the treatment plan adapts an evolutionary strategy that is able to mutate the shot number and size. For a big tumor with irregular shape, multiple initial treatment plans with different number of shot and shot size are generated via our medial axis guided algorithm. ${ }^{8}$ These plans evolve and compete as potentially optimal plans within the GESA analysis.

\subsection{Medial Axis GESA Seeding}

Medial axis detection is an image processing technique that is used to model the geometrical shape of an object of interest. The medial axis is a line representation of an object where all points are equidistant from at least two points along the object's border. One means of detecting the depth of voxels from the surface of the segmented tumor is the Grassfire Based Euclidean Distance Transform. A Euclid Distance Transform (EDT) of a binary image can be simulated as the propagation of the grass fire wave front. The fire is ignited all about the object's periphery and is extinguished at the innermost area where convergent fires create the highest local heat value. A sequential algorithm, based on Danielsson's work ${ }^{3,8}$ efficiently produces the EDT. First, distance values are assigned to the boundary points (i.e., at least one of the 8connected neighbors is a member of non-object), the fire source of the binary image object. These values are assigned by:

$$
\operatorname{EDM}(p)=\min \left[L\left(p, p^{\prime}\right)+E D M\left(p^{\prime}\right)\right] \quad \forall p^{\prime} \in N \cap S^{\prime}
$$

where $\mathrm{O}$ is the object, $\mathrm{O}^{\prime}$ is the non-object, $\mathrm{p} \in \mathrm{O}, \mathrm{p} \in \mathrm{O}^{\prime}, \mathrm{N}$ is the point set of 8 connected neighbors of $\mathrm{p}, \mathrm{L}$ is the real distance between $\mathrm{p}$ and $\mathrm{p}^{\prime}$. Next, the binary object is peeled away, one layer at a time. Old boundary points become members of a non-object, and new boundary points are exposed. For each set of new boundary points $\mathrm{p}$, the distance values are also calculated by Eq. 2. This calculation continues until it reaches the final layer of the object. The accuracy of grass fire-based EDT is improved by using a floating value instead of an integer value. The result better approximates a binary segmented object in digitized space.

Medial axis points may be detected as ridge points on an EDM surface via our Gradient Phase Operator. ${ }^{8}$ We define the phase gradient of a point on a 3D EDM surface:

$$
G(S, r, \varphi)=(E D M(S)-E D M(P)) / \Delta L
$$

where $\mathrm{S}$ is a point of the object, $\mathrm{P}$ is a point on a circle centered at $\mathrm{S}$ with radius of $\mathrm{r}$, $\varphi$ is the angle between line SP and the vertical direction, and $\Delta \mathrm{L}$ is the real distance between $\mathrm{S}$ and $\mathrm{P}$. The medial axis is an optimal location for LGK shot centroids.

\subsection{GESA Optimization}

Whether randomly or medial axis-generated, once seeded with 20 initial treatment plan families, the evolution process toward an optimal plan occurs automatically. 
Given the shape and volume of the tumor, the treatment planning team determines maximum shot number, initially uniform shot size. Then shot weight $(w)$ and position $(\operatorname{pos}[\mathrm{i}, \mathrm{j}, \mathrm{k}])$ are chosen randomly in the 20 initial plan domains, where $w \in[0.2,1.5]$, and pos $\in$ tumor voxel\}. For each family, twenty children are reproduced, each slightly mutated from its parent (Equation 4). Note that the reproduction the size of each shot can also mutate. If the weight of the shot falls under 0.2 , the shot size will change to the next smaller available size. Shot weight is assigned:

$$
w_{\text {mutated }}=w_{\text {current }} \times \frac{\text { size }_{\text {current }}}{\text { size } e_{\text {mutated }}}
$$

A $4 \mathrm{~mm}$ shot with weight below 0.2 would be removed from the treatment plan to reduce redundancy, thus change the total number of shots in the treatment plan. The initialized families are sent to GESA. After evaluating the fitness of each child (Equation 3) in each family, the best child competes with its parent for the parent position of the next generation based on Equation 5. Competition at the inter-family level results in replacement of parents of the worst performing family by that with the best treatment plan. This ensures that a family with the highest quality gets more chances to reproduce. This evolutionary procedure continues until the parents of each family change little (i.e., an objective function reaches an acceptable value).

\begin{tabular}{ccccccc}
\hline $\begin{array}{c}\text { Plan } \\
\text { Origin }\end{array}$ & $\begin{array}{c}\text { Tumor } \\
\text { Tiss. } \\
\text { Vol. }\end{array}$ & $\begin{array}{c}\text { Normal Tiss. } \\
\text { Vol. (Critical } \\
\text { Structure) }\end{array}$ & Shot \# & $\begin{array}{c}\text { Tumor } \\
\text { Dose } \\
\text { Avg }\end{array}$ & $\begin{array}{c}\text { Tumor } \\
\text { Dose } \\
\text { Std. Dev }\end{array}$ & $\begin{array}{c}\text { Planning } \\
\text { Time }\end{array}$ \\
\hline \multicolumn{7}{c}{ CASE 1: Acoustic Tumor } \\
\hline Manual & $6.69 \mathrm{~cm}^{3}$ & 2.01 & 8 & $0.62 D_{\text {pmax }}$ & 0.09 & 45 min \\
GESA & $6.77 \mathrm{~cm}^{3}$ & 1.32 & 7 & $0.65 D_{\text {pmax }}$ & 0.08 & 1 hour \\
\hline \multicolumn{7}{c}{ CASE 2: Meningioma Tumor } \\
\hline Manual & $5.40 \mathrm{~cm}^{3}$ & $4.77(0.03)$ & 19 & $0.60 \mathrm{D}_{\text {pmax }}$ & 0.10 & $5.5 \mathrm{hrs}$ \\
GESA & $5.47 \mathrm{~cm}^{3}$ & $3.16(0.02)$ & 15 & $0.62 \mathrm{D}_{\text {pmax }}$ & 0.11 & $7.5 \mathrm{hrs}$ \\
\hline
\end{tabular}

Table 1. Manual Plan and Optimized Plans for Cases 1 and 2.

\section{Brain Tumor Treatment Planning Experiment}

Our goal is to obtain an optimal treatment plan via GESA in less than one work day's ( 8 hours) computation time. We present two of the cases that test the effectiveness of our GESA treatment planning algorithm, especially where initial seeding is helpful. 
A

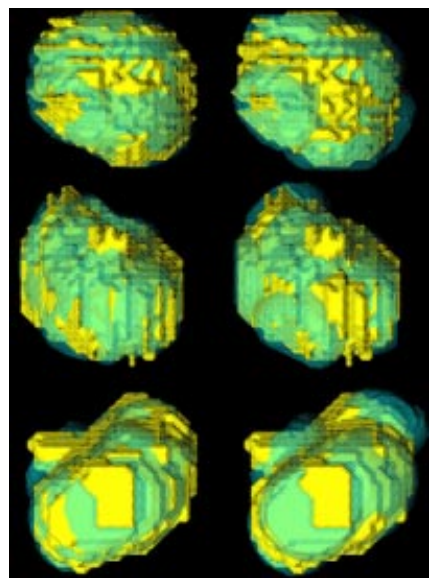

C

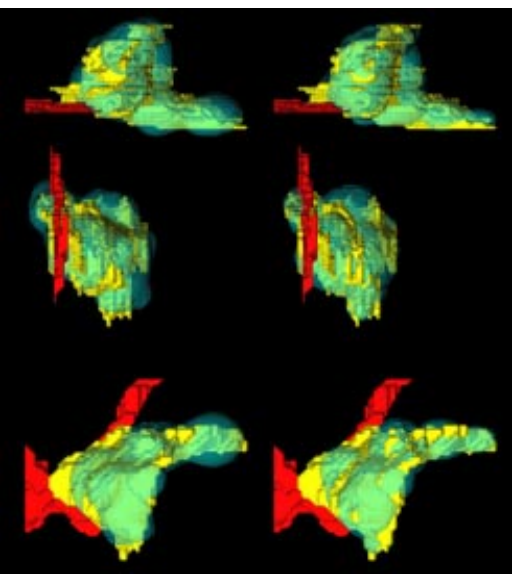

Figure 2. Comparison of Acoustic Tumor treatment plan (Columns A and B) and Meningioma Tumor treatment plan (Columns C and D) generated by GESA algorithm (Columns A and C) and that manually generated by a treatment planning team (Columns B and D). Views: Sagittal (Row 1), Coronal (Row 2), Axial (Row 3). The original tumor surface is opaque yellow. The surface of the volume receiving than $50 \%$ of $\mathrm{D}_{\text {pmax }}$ is assigned transparent blue.

\subsection{Case 1: A Small Acoustic Tumor with Simple Shape}

After determining the tumor volume, the attending treatment planning team set the initial shot number at 8 utilizing uniform shot size of $14 \mathrm{~mm}$, and uniform shot weight of 0.8 . The GESA began its search for a treatment plan with random shot location (i.e., no seeding). After 38 generations, a 7-shot treatment plan was generated (Table 1). The GESA treatment plan improved conformality (Figure 2) as evidenced by $0.08 \mathrm{~cm}^{3}$ more tumor irradiation, and $0.69 \mathrm{~cm}^{3}$ more normal tissue spared. The GESA optimized plan required 1 less shot and resulted in a higher tumor dose average which was closer to the desired dose (0.8), and a lower tumor dose standard deviation between voxels (i.e.,. homogeneity). over the manual shot packing plan (Figure 1). It took 1 hour to process the 38 GESA generations involved on a Silicon Graphics Inc. (Mountain View, CA) ORIGIN 200 ${ }^{\mathrm{TM}}$ SGI workstation with an R10000, $250 \mathrm{MHz}$ CPU. This unsupervised computation may be compared to the treatment planning team's 45 minutes spent manually generating the plan that the patient received.

\subsection{Case 2: A Large Meningioma Tumor with Complex Shape}

To initiate the GESA algorithm, the tumor's medial axis was located for a segmented meningioma tumor. Seven treatment plans with various shot number and shot size were based on the medial axis and provided as seeds to the GESA algorithm. Each of these seeds resulted in six families, each with twenty children. After 58 generations, the species with 15 shots that includes three $14 \mathrm{~mm}$-shots, nine $8 \mathrm{~mm}$-shots and three 
$4 \mathrm{~mm}$-shots won the competition. The randomized initial seeding required 214 generations (days) for GESA to reach an acceptable treatment plan. Table 1 shows the comparison between the plan generated by GESA and that generated by the planning team. GESA algorithm achieved better conformality and similar homogeneity. It took 7.5 hours to process the 58 GESA generations on the same SGI ORIGIN $200^{\mathrm{TM}}$. This unsupervised computation should be compared to the treatment planning team's requirement of 5.5 hours to manually generate their plan. Movies of the treatment plans seen in Figure 2 may be found at http://neurosurgery.cwru.edu/imaging.

\section{Discussion and Conclusion}

The 3D GESA algorithm searches the shot parameter space more thoroughly than is possible during manual shot packing. We note that, where beneficial to the patient, a reduction of shot number reduces treatment time. Also, as tumor shape becomes larger and/or more irregular the optimization search space of shot position, size, and weight expands exponentially. Medial axis seeding reduces that space by providing several good initial shot packing plans.

\section{References}

1. Shu, H., Yan, Y., Luo, L., Bao, X.: Three-dimensional optimization of treatment planning for gamma unit treatment system. Med. Phys., 25 (1998) 2352-2357.

2. Gibon, D., Rousseau, J., Castelain, B., Blond, S., Vasseur, C., Marchandise, X.: Treatment Planning Optimization by Conjugate Gradients and Simulated Annealing Methods in Stereotactic Radiosurgery. Int. J. Radiation Oncology Biol. Phys. 33 (1995) 201-210.

3. Wu, Q.J., and J. D Bourland "Morphology-guided radiosurgery treatment planning and optimization for multiple isocenters", Med. Phys. 26, October, pp. 2151-2160, 1999.

4. Wagner, T.H., Taeil, M.E., Ma, S.F., Meeks, S.L., Bova, F.J., Brechner, B.L., Chen, Y., Buatti, J.M., Friedman, W.A., Foote, K.D., Bouchet, L.G., A Geometrically Based Method for Automated Radiosurgery Planning. Int. J. Radiation Oncology Biol. Phys., 48 (2000) 1599-1611.

5. Leichtman, G.S., Aita, A.L., Goldman, H.W.: Automated Gamma Knife dose planning using polygon clipping and adaptive simulated annealing. Med. Phys., 27 (2000) 154-162.

6. Yip, P.P.C., Pao, Y.: Combinatorial Optimization with Use of Guided Evolutionary Simulated Annealing", IEEE Trans. Neural Networks, 6 (1995) 290-295.

7. Zhang, P., D. Dean, A. Metzger, and C. Sibata, "Optimization of Gamma Knife Treatment Planning via Guided Evolutionary Simulated Annealing”, Med. Phys., 28 (2001), in press.

8. Dean D, Metzger A, Duerk J, Kapur V, Zhang P, Chou H, Sibata D, and Wu J: Accuracy and Precision of Gamma Knife Procedure Planning and Outcomes Assessment. Computer Aided Surgery. 5 (1999) 63. 